\title{
Highly Angular Tolerant Transmission Filters for Narrow-Band Image Sensors
}

\author{
Fatima Omeis, Mondher Besbes, Christophe Sauvan, Henri Benisty* \\ Laboratoire Charles Fabry, Institut d’Optique Graduate School, Université Paris Saclay, CNRS, Palaiseau, France \\ Email: ^henri.benisty@institutoptique.fr
}

How to cite this paper: Omeis, F., Besbes, M., Sauvan, C. and Benisty, H. (2021) Highly Angular Tolerant Transmission Filters for Narrow-Band Image Sensors. Optics and Photonics Journal, 11, 140-151.

https://doi.org/10.4236/opj.2021.116012

Received: May 28, 2021

Accepted: June 26, 2021

Published: June 29, 2021

Copyright $\odot 2021$ by author(s) and Scientific Research Publishing Inc. This work is licensed under the Creative Commons Attribution International License (CC BY 4.0).

http://creativecommons.org/licenses/by/4.0/

\begin{abstract}
A dielectric transmittance filter composed of subwavelength grating sandwiched between two few-layers distributed Bragg reflectors (DBRs) is proposed with the aim of being compatible with CMOS technology and to be tunable by lithographic means of the grating pattern without the need of thickness changes, in the broad spirit of metamaterials. The DBR mirrors form a Fabry-Perot (FP) cavity whose resonant frequency can be tuned by changing the effective refractive index of the cavity, here, by tailoring the in-plane filling factor of the grating. The structure has been studied and designed by performing numerical simulations using Fourier Modal Method (FMM). This filter proves to have high broad angular tolerance up to $\pm 30^{\circ}$. This feature is crucial for evaluating the spectral performance of narrow-band filters especially the so-called Ambient light sensors (ALS). By analyzing the transmittance spectral distributions in the band diagram, it is found that the angular tolerance is due to coupling between the FP and the guided mode inside the cavity in analogy to resonances occurring within multimode periodic waveguides in a different context.
\end{abstract}

\section{Keywords}

Narrow-Band Filters, Transmission, CMOS, Angular Tolerant, Subwavelength Grating, Distributed Bragg Reflectors (DBR), Fabry-Perot Cavity

\section{Introduction}

Driven by the increased use of smart devices such as mobile phones and compact cameras, rapid growth in digital color imaging devices is observed [1] [2]. These devices rely on the use of complementary metal oxide semiconductor (CMOS) arrayed image sensors exploiting pigmented and dye-based color filters 
such as RGB (Red-Green-Blue) Bayer-filters [3] for eye vision. There are three challenging aspects for these filter technologies [4]: 1) to go to robust materials, such as ultraviolet-robust ones; 2 ) to ease mass fabrication, by changing in-plane nanostructure parameters rather than sequentially depositing numerous filters; 3 ) to perform better or new photonic functions, such as narrow-band and/or highly angular-tolerant filtering. This last point is the main motivation of this paper, especially for (ALS) sensors [5]. While these devices in the most common version just capture ambient light to correct smartphone displays, the present study rather addresses the more advanced versions that have multispectral capability (e.g. IR and UV added in the ST Microelectronics VD6281 product, see information on product datasheet) that generically aim at capturing the spectral content of a scene with better resolution than the usual RGB triple (and of course monochrome detectors). They are used to optimize scene rendering for a variety of situations (i.e., indoors or outdoors) for people's faces or for goods displayed on websites. They can also be envisioned for all kinds of environment colorimetric sensing, obviously enough.

For filter robustness, as the resolution is improved, it is observed that the decreased pixel area translates into larger degradation under ultraviolet illumination or high temperature environments and weaken efficiency. To address this issue, nanostructured surfaces can be used such as plasmonic filters which are based on the use of thin films or patterned metallic (i.e., $\mathrm{Au}, \mathrm{Ag}$, or $\mathrm{Al}$ ) nanostructures that exhibit large tunability over the visible spectral range [6] [7] [8]. They also offer the opportunity of single rather than multiple deposition steps. While plasmonic filters might appear as a solution, their efficiency is relatively low due to the significant optical losses of metals in the visible range, and their fabrication procedure is not compatible with CMOS technology, making them a weak competitor for industrialists.

On the other hand, subwavelength gratings based on dielectric materials have lower intrinsic losses, yielding color filters with higher luminosity [9]. This makes dielectric structures most suitable to improve the spectral features of CMOS image sensor devices in terms of resonant behavior and tunability [10] [11]. By varying the geometry of the dielectric nanostructures especially in the plane array, the position of the resonances can be tuned throughout the whole visible and infrared range [12]. Many studies were carried out to understand and fabricate dielectric and metal-based spectral filters possibly with angular insensitive properties [13] [14] but with a focus on highly efficient reflection.

Thus, the fabrication and analysis of high transmission spectral filters, to our knowledge [15] [16], has not provided many answers on the issue of angle of incidence impact on the optical response, especially in the demanding spectral context of ALS sensors. Indeed, these optical, material and geometrical considerations are linked to the type of the desired filtering. For the RGB case, the spectral width is comfortably large, say $60-100 \mathrm{~nm}$. But for ALS of the forthcoming generation (with more resolution than basic ones, which operate not much beyond RGB selection), or for wider applications aiming for instance at 
getting agro-forestry and field data on various stresses through leaves reflectance, finer resolution (i.e., $15-30 \mathrm{~nm}$ ) of the filter is needed. That makes the angular dependence of the transmission filter even more of an issue.

The most common way to design a single transmission optical filter is to form an FP cavity using a pair of reflective mirrors [17], the dielectric version of which are the well-known (DBRs) periodic stacks [4] [18]. However a different filter is needed for each spectral band. Thus a nanostructured version, offering the lithographic-feasibility advantages as mentioned above, is the most welcome. In this work, it is demonstrated that tunability and high angular tolerance are achieved in such structures using a high-index grating embedded between two dielectric DBRs. The geometrical parameters of the structures are optimized so that an angular insensitivity up to $30^{\circ}$ is achieved, while preserving a substantial degree of lithographic tunability in line with the advanced ALS application.

\section{Structure and Design}

The targeted design of the FP filter with embedded dielectric structure is shown schematically in Figure 1(a), it consists of two high-reflectivity DBR mirrors and a high-index grating inside. Each mirror is a set of 3 pairs of quarter wavelength layers made of very well-mastered materials, silicon nitride $\left(\mathrm{Si}_{x} \mathrm{~N}_{y}\right.$ with $y: x \simeq 4: 3, \mathrm{SiN}$ in short, of index $\left.n_{H}=2\right)$ and silicon oxide $\left(\mathrm{SiO}_{2}\right.$, $\left.n_{L}=1.456\right)$. The central wavelength of the DBRs is $\lambda_{0}=700 \mathrm{~nm}$. The grating layer (period $d=300 \mathrm{~nm}$, and height $h_{r}=100 \mathrm{~nm}$ ) is centered between the 2 DBRs and encapsulated in $\mathrm{SiO}_{2}$ material with a thickness $t_{c}=100 \mathrm{~nm}$ on each side.

The refractive index of the grating is chosen to be $n_{r}=4$, which is an approximated value of that of amorphous silicon (a:Si) in the considered spectral range. The use of high refractive indices entails some semiconductor-type aboveband-edge absorption (albeit with a smooth edge for a:Si), a likely reason why a $\mathrm{DBR}+$ cavity solution purely based on those materials is not commonly proposed. The grating thus offers the advantage to limit the impact of absorption, especially when the relative fraction of a:Si is small, say $w / d \lesssim 0.2$, which corresponds to the smaller average grating index and thus to shorter wavelengths that are more at risk of absorption in such materials. The numerical simulation of the optical response of the structure (reflection, transmission and electricfield distribution) is done using FMM (also known as Rigorous Coupled Wave Analysis RCWA) implemented with the S-matrix algorithm [19] [20]. For a conventional DBR mirror, composed of 6 pairs of alternating $\mathrm{SiO}_{2}-\mathrm{SiN}$ dielectric layers, the high reflectivity of light is caused by the constructive interference between incident and reflected light due to Fresnel reflections. This will appear as a forbidden band of transmission centered at $700 \mathrm{~nm}$, Figure 1(b). An FP cavity is formed when a defect is introduced in the DBR mirror. This results in a Lorentzian-shape transmission peak (Airy function resonance) at a specific wavelength $\lambda_{c}$ that in a simple model reads: 

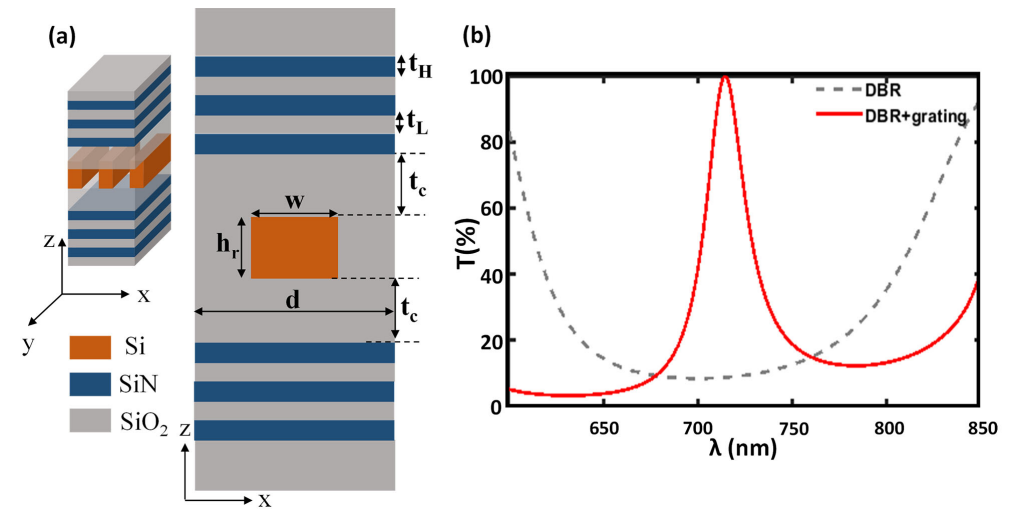

Figure 1. (a) Structure of a transmissive narrow-band filter formed of a grating layer between 2 DBRs. Both outer media are $\mathrm{SiO}_{2}$. (b) Comparison of transmission spectra: The dashed line is the sole set of two DBR mirrors (no spacer) with its $\sim 200 \mathrm{~nm}$ stopband. The red solid line resonant at $715 \mathrm{~nm}$ is for the structure shown in (a), two DBRs with a grating layer.

$$
2 n_{c} L_{c}=m \lambda_{c}
$$

where $L_{c}$ is the thickness of the entire cavity, $n_{c}$ the adequate average refractive index of the cavity medium, and $m$ the integer order of the resonant mode [21]. For a grating width $w=270 \mathrm{~nm}$, and a spacing $t_{c}=100 \mathrm{~nm}$ on each side of the grating, a transmission peak appears within the stopband of the DBR at $\lambda_{c}=715 \mathrm{~nm}$. The position of the transmission peak can be tailored by modifying the optical length of the cavity. This can be achieved by changing the filling factor $f=w / d$ of the grating and thus the effective index of the structured layer. Figure 2 shows simulated passbands for six different grating widths $w$ varying between 90 and $295 \mathrm{~nm}$, and exhibiting a transmission peak up to $100 \%$ and a full width at half maximum (FWHM) around $20-30 \mathrm{~nm}$. The resonant wavelengths of the passbands vary from $635 \mathrm{~nm}$ to $725 \mathrm{~nm}$. These numbers are typical of the demands for ALS sensors. This is logical with the effective index increasing gently with $w$ as follows from effective medium theory with the electric field in the $y$ direction (TE polarization), meaning that the dielectric constant ( $\varepsilon=n^{2}$ ) would be plainly averaged in the deep subwavelength regime.

On this apparently simple basis, the present study tackles the issue of angular dependence as follows. Figure 3 displays the transmission as a function of the frequency (normalized as $u=d / \lambda=\omega d /(2 \pi c)$ ) and the wavevector along the grating periodicity axis, $k_{\|}=k_{x}$. Two main features can be seen: 1) FP resonances located at $u_{c}=d / \lambda_{c}$ in the DBR bandgap; and 2) guided-modes resonances resulting from the excitation of guided modes of the whole system. Note that there may be several FP resonances if several values of $m$ fall in the DBR bandgap, but a single one is a preferred choice. Neglecting periodicity, guided modes fold themselves at the edge of the first Brillouin zone $\left(k_{x}=\pi / d\right)$, and come back to $k_{x}=0$ with a gentle and regular dispersion slope, assuming for the design step that the group index dispersion $n_{g}(\omega)$ of all concerned modes is modest. 


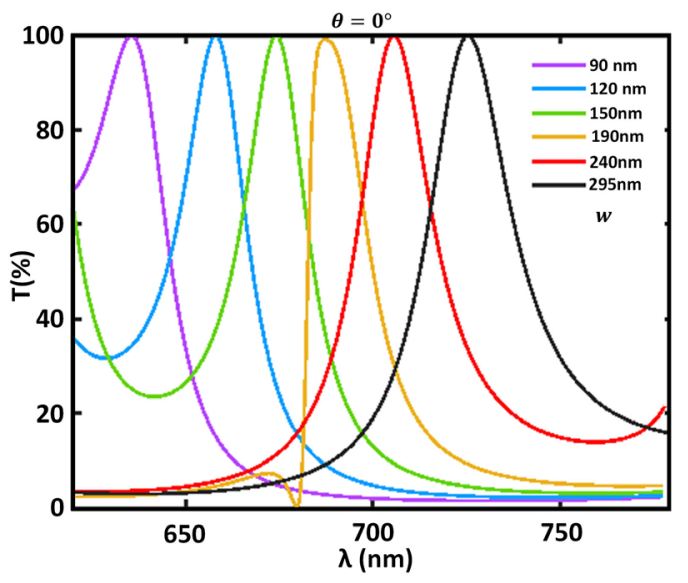

Figure 2. Simulated transmission spectra, with both outer media being $\mathrm{SiO}_{2}$, for six different widths $w$ of the grating layer ranging from 90 to $295 \mathrm{~nm}$. The transmission peak is above $99 \%$.

If such guided modes intersect with the FP resonances, however, a mode coupling effect occurs due to the periodic component of the dielectric landscape. This is quite analogous to mode coupling in photonic crystal waveguides [22] [23], with higher-order modes playing the role of FP resonances (minimal $k_{x}$ ) and lower order modes playing the role of the guided modes (having larger $k_{x}$ for the main field Fourier components, but folded). This is at variance with the usual case of resonant waveguide gratings, whereby the incident light has small $k_{x}$ but is a smooth continuum instead of being made resonant by the DBRs. The interaction, in any event, impacts the dispersion of both modes, FP and guided. Later on it is shown that one can take advantage of a positive impact of this coupling mechanism in terms of angular tolerance. Indeed, the modal interaction can counteract the usual angular trends of FP modes [24]. It is well-known that this basic dependence corresponds to a constant wavevector in the vertical direction, and results in $\lambda_{c}\left(\theta_{c}\right)=\lambda_{c}(0) \cos \left(\theta_{c}\right)$, where the angle is referenced in the cavity $\left(k_{x}=n_{c} \sin \left(\theta_{c}\right) 2 \pi / \lambda_{c}\right.$ giving the wavevector, more appropriate to deal with periodic structures). So if one deals with a resonance at $715 \mathrm{~nm}$ at normal incidence, a $30^{\circ}$ inner angle (relevant for CMOS sensors) incurs a factor $\cos \left(\theta_{c}\right) \simeq 0.866$ and thus a $\sim 95 \mathrm{~nm}$ blue shift to $620 \mathrm{~nm}$, rather detrimental for a filtering width targeting the range $20-50 \mathrm{~nm}$.

In the discussion part, a comparison is done between the angular tolerance obtained with the design having the kind of modal interaction described below (not showing the dispersion diagram in this short paper, though), to the naive FP result for silica spacer. Spacers with larger index would also be meaningful comparisons, but consider that the change from silica to SiN brings only a factor $\sim 4 / 3$ on the index, while a:Si is not desirable in large thicknesses for shorter wavelength.

\section{Results and Discussion}

For the most classical multilayer FP filters, the wavelength center varies with the angle of incidence, in the form of a quadratic blue shift [25]. For a textbook FP 
cavity, the determining factor is the cavity refractive index, that intervenes as $1 / n^{2}$. As for guided mode resonance filters in the context of array sensors, studies showed that reflective color filters based on this technique are of high efficiency (high transmission and good rejection), but they are quite poor in tolerating the angular incidence [26], with a linear wavelength shift in basic designs. Improved design involving two-dimensional structures showed good angular independence of reflection in the visible range [14], but only for a broader range corresponding to a whole band in the band structure. Therefore, given these trends, there is no clear design approach that addresses all three aspects of 1) narrowband transmission (FP-type); 2) tunabilty by lithography (as done in resonant waveguide grating); and 3) good angular tolerance. Thus, the angular tolerance is primarily examined in the results, while considering also how multi-band spectral structure performs for different filling factors.

To illustrate clearly the effect of the incident angle on the optical response of the structure, optical transmission $T\left(k_{\|}, \omega\right)$ in the form of band diagrams is presented for TE polarization as shown in Figure 3(a) where $k_{\|}=k \sin \theta$ is the in-plane wave vector with $\theta$ the incident angle in silica and $k$ the incident wave vector also in silica. Let us consider a specific structure of definite geometrical parameters $\left(d=300 \mathrm{~nm}, w=150 \mathrm{~nm}, h_{r}=100 \mathrm{~nm}\right.$, and $\left.t_{c}=100 \mathrm{~nm}\right)$. The stopband of the DBRs shows up between $d / \lambda=0.36$ and 0.49 , where no light is transmitted except for the passbands created by the \{spacer + grating layer\} forming the FP cavity. As the incident angle increases, the transmission spectrum maintains almost a straight curve until $30^{\circ}$. To better understand the optical behaviour of the structure the electric field (EF) distribution in the $x z$ plane is studied. Figure $3(\mathrm{~b})$ illustrates the electric field profile in TE polarization for normal incidence and at $30^{\circ}$. One finds that at normal incidence, two transmission peaks appear, labelled by 1 and 3 respectively. Peak 1 is the resonant wavelength of interest that is tuned by the grating width $w$. It corresponds to the second order FP mode (using as $L_{c}$ in the simple model the distance between the SiN inner layers for simplicity); its field is concentrated at the top and bottom edges of the high index "atom", whereas in a planar case the two maxima of a 2 nd order FP mode would have horizontal phase fronts. Peak 3 is located close to the high frequency edge of the DBR stopband. Here, the EF pattern appears as a huge hybridization of an FP mode of even vertical parity (whereas Peak 1's EF had odd vertical parity) with a folded guided mode having one antinode squeezed in the high index and the adjacent antinodes spanning the silica cavity. At $30^{\circ}$ incidence in air, the light line, in red, intersects the transmission diagram at two resonances marked as peak 2 and peak 4 . Peak 2 is the edge of the extension of peak 1 resonance, with less than $1 \%$ shift from the $0^{\circ}$ frequency, thus a resonance highly tolerant to the incident angle (more on that later). It is clear that at this peak the EF is still concentrated at edges of the high index "atom" while the EF in the DBRs shows the hybridization of the FP mode with higher-order guided modes seen as steep streaks in Figure 3(a) with faster EF vertical decay. 


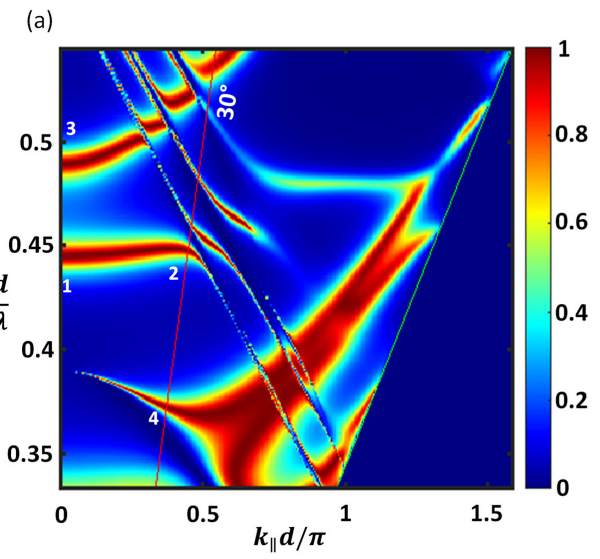

(b)

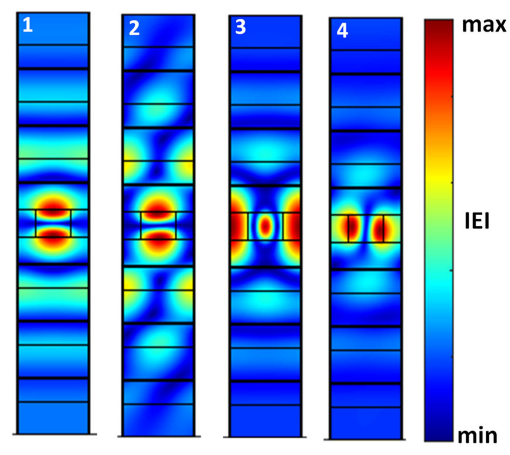

Figure 3. (a) Transmission map calculated by FMM. The red line corresponds to $30^{\circ}$ in air and the green line is the light-line $\left(90^{\circ}\right.$ in silica). (b) Electric-field profile in the structure for a TE polarized incident plane wave, $(1-3)$ at normal incidence, $(2-4)$ at $30^{\circ}$ incident angle.

The anticrossings of the FP modes with these modes are responsible for flattening out the FP mode in the cavity hence resulting in the highly improved angular tolerance compared to more parabolic FP modes in silica. As for peak 4, its field clearly shows that it is a horizontally propagating guided mode minimally spreading in the innermost SiN DBR layers. Due to orthogonality with the branch from peak 3 , its antinodes are at the vertical edges of the "atom" (in other words, branch 3 field keeps a similar nearly even pattern, so peak 4 is nearly odd) with a noticeable distortion due to the oblique incidence.

From this general analysis in a band-diagram spirit, the design rules for filters can be greatly anticipated. For instance, the extreme frequencies are first bounded by the DBR stopband, but also, the period plays a role in locating adequately the DBR-induced guided modes (steep streaks) such that the anticrossing can play the expected flattening role for the band angular $\left(k_{\|}\right)$behavior of the filter lines.

For the purpose of finely evaluating the optical performance of our structure, the transmission contour is plotted for three different filling factors ( $f=0.5,0.75$ and 0.99) corresponding to the blue, green, and red lines respectively in Figure 4 (note that the frequencies are not corresponding to the classical RGB filters here, since this study deals with much narrower and closer spectral lines). The shaded regions correspond to the areas of near-maximal transmission at $T \geq 0.9$. Their shape shows where the risks of large cross-talk take place in terms of angle and wavelength. It is seen for instance that large angles of the lower resonance (in red) nearly touch at $d / \lambda \simeq 0.425$ the bottom of the upper resonance hightransmission area of the upper resonance (in green).

It is now appropriate to study the device-oriented filtering performance. For this purpose, an additional calculation is conducted and the results are averaged on typically 100 uniformly distributed angles. The right side of the panel shows the transmission spectra for the three filling factors. The solid lines are calculated 


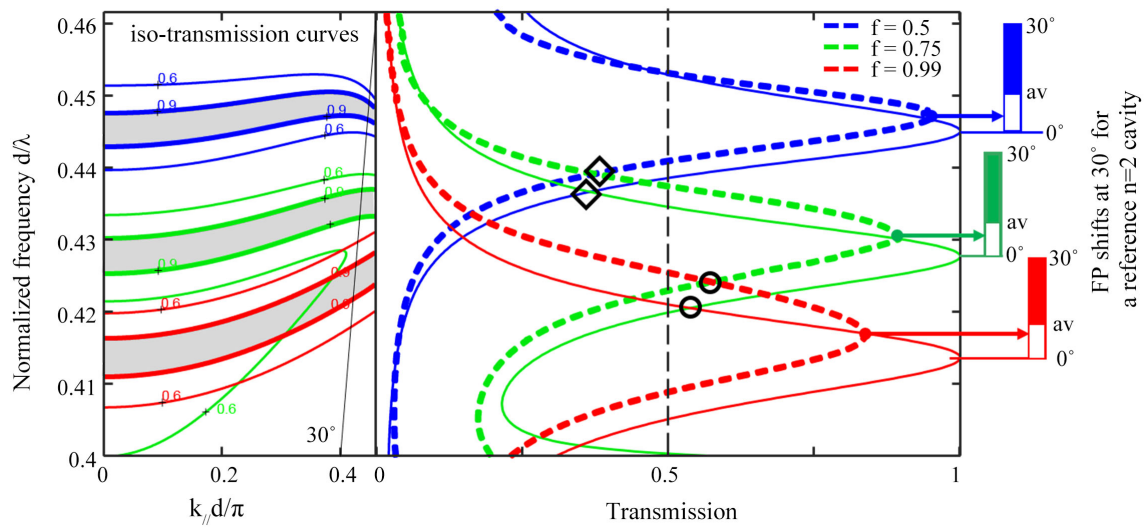

Figure 4. (Left side) Transmission contours (iso-transmission curves of the wavevectorfrequency map) calculated by FMM for three different filling factors $f=0.5,0.75$ and 0.95 (blue, green, red lines), with a gray shade for the highest transmission area (contours are $T=0.6$ and $T=0.9$, this latter with a thicker line). (Right side) Transmission spectra corresponding to the three filling factors, at $0^{\circ}$ the straight curve, and the angle-average of the transmission spectra for each filling factor over the $0^{\circ}$ to $30^{\circ}$ range in air. The small open disks help tracking the red-green intersection and the small open diamonds the green-blue intersection, modestly impacted by angle-induced broadening. The bars on the right outside are intended to compare the results to the angle-induced spans for ideal FP cavities with spacer index $n=2$, with the average over the $30^{\circ}$ span marked by the hollow part (av). The arrows show how much our design performs better than such a reference cavity.

spectra at normal incidence, while the dashed curves are the calculated average transmission over the angular interval that spans from $0^{\circ}$ to $30^{\circ}$ with angles taken here in air, $k_{\|}=2 \pi \sin (\theta) / \lambda$. It is clear that the tuning procedure is not much affected by the angular dependency of the structure causing here a slight shift in the resonant frequency between the normal incidence and the average transmission response. It is worth noting that the average shape of the resonance is almost unchanged as regards the span of frequency at $T=0.5$, undergoing only a rigid blue shift. It is only because the maxima are lower that the width at half-maximum is slightly larger. Intersections of red and green dashed curves take place at $\sim 60 \%-65 \%$ of maximum instead of $50 \%$ for the solid lines (open disks). For the blue-green intersection (open diamonds), the shift is only from $\sim 30 \%$ to $\sim 40 \%$ of maximum.

Moreover, to highlight the interest and the success of the strategy, we compare the flattened angular shift of the structure with that of a canonical FP with ideal mirrors and an $n=2$ spacer, thus following for its frequency shift with angle the textbook trend $\left(\cos \theta^{\prime}\right)^{-1}$ trend (where $\theta^{\prime}$ is the angle in the spacer medium, hence $\left.\left(\cos \theta^{\prime}\right)^{-1} \simeq[\cos (\theta / 2)]^{-1} \simeq 1+\theta^{2} / 8\right)$. In our cavity, a major part of the field samples silica areas of lower (and thus less favorable) index, only a modest part of the field samples the larger "atom" index. So this comparison is a fair one to evaluate the success of our strategy. The bars indicate the frequency shift for a $30^{\circ}$ span (in air) and the white part inside the bar indicates the average (essentially located at $1 / 3$ of the span given the quasi-parabolic shift of the ca- 
nonical FP model). For the red case, our design is marginally better (vs. the demanding benchmark mentioned, however), while it becomes about twice better for the blue case, which is flatter due to the stronger interaction with the guided modes. Hence, this design can typically provide a set of adjacent filters with at most $4 \%$ relative spectral width, and with an angular tolerance up to $30^{\circ}$ in air. Let us remind that usual RGB filters (Bayer's or others) have a relative width around $20 \%$, a lot more relaxed, but colorimetric requirements may then be raised to higher standards, as witnessed by the large literature hinted in the introduction.

Specifying an accurate value for the angular tolerance depends on the limits set to the cross-talk among the desired spectral channels, with the definition of the cross-talk being also application-specific as the ALS sensor products and the related applications are not stabilized yet.

It is now instructive to see how the proposed strategy can be applied to different kinds of arrayed or small-size sensors that are mass produced in the CMOS industry. For contemporary camera sensors, with commonly than $10 \mathrm{M}$ pixels, the pixel size is around 2 microns, and Bayer filters are the general solution. Then, it is delicate to fully conclude about the proposed grating approach because of finite-size effect: firstly some lateral photon transport is inherent to this strategy, secondly, if there are less than 10 periods, say, it means that the bands are correspondingly blurred in the dispersion diagram, and that the transmission can be perturbed to a sizable extent. This would demand further studies, possibly taking advantage of existing studies on resonant waveguide grating to retain the desired effects at small pixel sizes.

On the other hand, the emerging ALS devices operate at much bigger sensor sizes, in the range 100 to 500 microns. At such sizes, the proposed design would have hundreds of periods and the size-effect is expected to be negligible. The trend in ALS should keep those sizes large even in case of further integration, because ALS are expected to produce signals quickly, at the millisecond time scale, in order to provide immediate correction to the exposure of the main image sensor. For a given scene, the photon flux in a pixel involves much longer exposure times for sensor sizes of a few microns (say, $20 \mathrm{~ms}$ or more), whereas the same scene may naturally provide a large signal-to-noise ratio after, say, $2 \mathrm{~ms}$, because of the larger unit sensor area, by a factor $\sim 10^{4}$. Overall, the proposed filter strategy should be viable for the whole next generation of ALS devices, either for smartphones or for a variety of niche diagnostic applications suggested above.

\section{Conclusions}

In summary, this paper proposes a CMOS-compatible narrow-band transmission filter that consists of a high-index one-dimensional grating layer sandwiched between two DBR mirrors. In line with the effective-index approach, the studied structure proves to be tunable by adjusting only the lateral width of the grating "atom", avoiding the need to change film thicknesses. The transmission 
map $T\left(k_{\|}, \omega\right)$, akin to a band diagram, was calculated using the FMM method. The electric field distribution of the resonant modes and the role of field concentration at the "atom" edge was discussed, underlining the interplay of vertical Fabry-Perot resonance and horizontal guidance. The main applied scope of the study was the improvement of narrow-band type ALS sensors, in terms of angular tolerance and tunability. Narrow bands are helpful to fine-tune color rendering in various situations, and could also help cheaper monitoring of colorimetric diagnostics. They must feature high yield to react adjust camera setting at faster rates than the frame rate even in a low-light environment, meaning that a high peak transmission is mandatory.

By properly adjusting the position of the different resonances, high angular tolerance is obtained across the different channels associated with the different filling factors. An appropriate benchmark consists of comparing it to the frequency shift in an ideal FP cavity with $n=2$. It is shown that the design can tolerate oblique incidences up to $30^{\circ}$ (in air) with a frequency blue-shift twice smaller than that of the benchmark cavity. Such improvement is crucial because, unlike the RGB filter counterpart with $\sim 20 \%$ spectral width, whose angular tolerance was much addressed, the bands are targeted here with only $\sim 4 \%$ spectral width, naturally much more demanding. To our knowledge, these enhanced properties and improved features have not been achieved for these optical functions in a CMOS-compatible and high-transmission setting, neither in the visible nor in the near infrared range. So understanding the angular influence on the optical performance of these narrowband filters is helpful to ameliorate the multispectral ALS sensors.

Further studies are needed for two-dimensional grating and polarizationdependence toward actual devices. The principles used here must be carefully handled to assess their role in these extensions. The role of fabrication constraints and lithographic accuracy can also be explored. The last direction is to consider the system aspects, in terms of a number of channels, expected crosstalk, and significance for, e.g. color rendering or diagnostic applications. This study assumed large devices with no edge effects, but applications to reduced sensor sizes with fewer grating periods could be an interesting challenge.

\section{Acknowledgements}

The authors would like to thank the STMicroelectronics and CEA colleagues of the Pixonano project (IPCEI program) in Crolles and Grenoble that provided the background of the present investigation: Sandrine Villenave, Frédéric Boeuf, Stéphane Monfray and Quentin Abadie.

\section{Conflicts of Interest}

The authors declare no conflicts of interest regarding the publication of this paper.

\section{References}

[1] Sharma, G. (2003) Digital Color Imaging Handbook. CRC Press, Boca Raton. 
[2] André, P.S., Nero, L., Freitas, V.T., Relvas, M.S. and Ferreira, R.A.S. (2013) Printable Optical Filters for Visible Optical Communications. Optics and Photonics Journal, 3, 136-138. https://doi.org/10.4236/opj.2013.32B033

[3] Choi, J., Sakong, C., Choi, J.H., Yoon, C. and Kim, J.P. (2011) Synthesis and Characterization of Some Perylene Dyes for Dye-Based LCD Color Filters. Dyes and Pigments, 90, 82-88. https://doi.org/10.1016/j.dyepig.2010.11.006

[4] Masarotto, L., Frey, L., Charles, M.L., Roule, A., Rodriguez G., Souil, R., Morales, C. and Larrey, V. (2017) Transmission Measurements of Multilayer Interference Filters Developed for a Full Integration on Complementary Metal Oxide Semiconductor Chips. Thin Solid Films, 631, 23-28. https://doi.org/10.1016/j.tsf.2017.03.055

[5] Junger, S., Verwaal, N., Tschekalinskij, W. and Weber, N. (2014) Near-Infrared Cut-Off Filters Based on CMOS Nanostructures for Ambient Light Sensors and Image Sensors. Proceeding of the OPTO 2014 Conference, Photonic and Phononic Properties of Engineered Nanostructures IV, Vol. 8994, San Francisco, 1-6 February 2014, Article No. 89941K. https://doi.org/10.1117/12.2039470

[6] Bibbò, L., Khan, K., Liu, Q., Lin, M., Wang, Q. and Ouyang, Z. (2017) Tunable Narrowband Antireflection Optical Filter with a Metasurface. Photonics Research, 5, 500-506. https://doi.org/10.1364/PRJ.5.000500

[7] Dai, P., Wang, Y., Hu, Y., de Groot, C.H., Muskens, O., Duan, H. and Huang, R. (2021) Accurate Inverse Design of Fabry-Perot-Cavity-Based Color Filters Far beyond sRGB via a Bidirectional Artificial Neural Network. Photonics Research, 9, B236-B246. https://doi.org/10.1364/PRJ.415141

[8] Kim, I., Yun, J., Badloe, T., Park, H., Seo, T., Yang, Y., Kim, J., Chung, Y. and Rho, J. (2020) Structural Color Switching with a Doped Indium-Gallium-Zinc-Oxide Semiconductor. Photonic Research, 8, 1409-1415. https://doi.org/10.1364/PRJ.395749

[9] Berzinš, J., Fasold, S., Pertsch, T., Bäumer, S. and Setzpfandt, F. (2019) Submicrometer Nanostructure-Based RGB Filters for CMOS Image Sensors. ACS Photonics, 6, 1018-1025. https://doi.org/10.1021/acsphotonics.9b00021

[10] Horie, Y., Han, S., Lee, J.-Y., Kim, J., Kim, Y., Arbabi, A., Shin, C., Shi, L., Arbabi, E., Kamali, S.M., Lee, H.-S., Hwang, S. and Faraon, A. (2017) Visible Wavelength Color Filters Using Dielectric Subwavelength Gratings for Backside-Illuminated CMOS Image Sensor Technologies. Nano Letters, 17, 3159-3164. https://doi.org/10.1021/acs.nanolett.7b00636

[11] Xu, Z., Li, N., Dong, Y., Fu, Y.H., Hu, T., Zhong, Q., Zhou, Y., Li, D., Zhu, S. and Singh, N. (2021) Metasurface-Based Subtractive Color Filter Fabricated on a 12-Inch Glass Wafer Using a CMOS Platform. Photonics Research, 9, 13-20. https://doi.org/10.1364/PRJ.404124

[12] McClung, A., Samudrala, S., Torfeh, M., Mansouree, M. and Arbabi, A. (2020) Snapshot Spectral Imaging with Parallel Metasystems. Science Advances, 6, Article No. eabc7646. https://doi.org/10.1126/sciadv.abc7646

[13] Yang, C., Hong, L., Shen, W., Zhang, Y., Liu, X. and Zhen, H. (2013) Design of Reflective Color Filters with High Angular Tolerance by Particle Swarm Optimization Method. Optics Express, 21, 9315-9323. https://doi.org/10.1364/OE.21.009315

[14] Cheong, B.-H., Prudnikov, O.N., Cho, E., Kim, H.-S., Yu, J., Cho, Y.-S., Choi, H.-Y. and Shin, S.T. (2009) High Angular Tolerant Color Filter Using Subwavelength Grating. Applied Physics Letters, 94, Article ID: 213104. https://doi.org/10.1063/1.3139058

[15] Stoevelaar, L.P., Berzinš, J., Silvestri, F., Fasold, S., Kamali, K.Z., Knopf, H., Eilen- 
berger, F., Setzpfandt, F., Pertsch, T. and Bäumer, S. and Gerini, G. (2020) Nanostructure-Modulated Planar High Spectral Resolution Spectro-Polarimeter. Optics Express, 28, 19818-19836. https://doi.org/10.1364/OE.392536

[16] Horie, Y., Arbabi, A., Arbabi, E., Kamali, S.M. and Faraon, A. (2016) Wide Bandwidth and High Resolution Planar Filter Array Based on DBR-Metasurface-DBR Structures. Optics Express, 24, 11677-11682. https://doi.org/10.1364/OE.24.011677

[17] Yue, W., Lee, S.-S. and Kim, E.-S. (2016) Angle-Tolerant Polarization-Tuned Color Filter Exploiting a Nanostructured Cavity. Optics Express, 24, 17115-17124. https://doi.org/10.1364/OE.24.017115

[18] Horsley, S.A.R., Wu, J.-H., Artoni, M. and La Rocca, G. (2014) Revisiting the Bragg Reflector to Illustrate Modern Developments in Optics. American Journal of Physics, 82, 206-213. https://doi.org/10.1119/1.4832436

[19] Moharam, M.G., Grann, E.B., Pommet, D.A. and Gaylord, T.K. (1995) Formulation for Stable and Efficient Implementation of the Rigorous Coupled-Wave Analysis of Binary Gratings. Journal of the Optical Society of America A, 12, 1068-1076. https://doi.org/10.1364/JOSAA.12.001068

[20] Li, L. (1996) Formulation and Comparison of Two Recursive Matrix Algorithms for Modeling Layered Diffraction Gratings. Journal of the Optical Society of America $A, 13,1024-1035$. https://doi.org/10.1364/JOSAA.13.001024

[21] Ismail, N., Kores, C.C., Geskus, D. and Pollnau, M. (2016) Fabry-Pérot Resonator: Spectral Line Shapes, Generic and Related Airy Distributions, Linewidths, Finesses, and Performance at Low or Frequency-Dependent Reflectivity. Optics Express, 24, 16366-16389. https://doi.org/10.1364/OE.24.016366

[22] Olivier, S., Rattier, M., Benisty, H., Weisbuch, C., Smith, C.J.M., De La Rue, R.M., Krauss, T.F., Oesterle, U. and Houdré, R. (2001) Mini-Stopbands of a OneDimensional System: The Channel Waveguide in a Two-Dimensional Photonic Crystal. Physical Review B, 63, Article ID: 113311. https://doi.org/10.1103/PhysRevB.63.113311

[23] Ayre, M., Cambournac, C., Khayam, O., Benisty, H., Stomeo, T. and Krauss, T.F. (2008) Photonic Crystal Waveguides for Coarse-Selectivity Devices. Photonics and Nanostructures-Fundamentals and Applications, 6 19-25. https://doi.org/10.1016/j.photonics.2007.09.004

[24] Khayam, O. and Benisty, H. (2009) General Recipe for Flatbands in Photonic Crystal Waveguides. Optics Express, 17, 14634-14648.

https://doi.org/10.1364/OE.17.014634

[25] Tikhonravov, A.V., Trubetskov, M.K., Amotchkina, T.V. and Yanshin, S.A. (2007) Design of Multilayer Coatings with Specific Angular Dependencies of Color Properties. In: Optical Interference Coatings, OSA Technical Digest (CD), Optical Society of America, Washington DC, WB2. https://doi.org/10.1364/OIC.2007.WB2

[26] Tibuleac, S. and Magnusson, R. (1997) Reflection and Transmission Guided-Mode Resonance Filters. Journal of the Optical Society of America A, 14, 1617-1626. https://doi.org/10.1364/JOSAA.14.001617 